\title{
Superoxide dismutases, lung function and bronchial responsiveness in a general population
}

\author{
M. Siedlinski*, C.C. van Diemen*, D.S. Postma\# , J.M. Vonk* and H.M. Boezen*
}

ABSTRACT: Oxidative stress is an important causative factor in the onset and progression of smoking-related lung diseases, such as chronic obstructive pulmonary disease (COPD). Superoxide dismutases (SODs) can prevent an increase in oxidative burden.

A total of 1,390 subjects from the prospective Vlagtwedde-Vlaardingen cohort were genotyped for two single nucleotide polymorphisms (SNPs) in SOD2 and four SNPs in SOD3, which were further analysed for associations with the presence of bronchial hyperresponsiveness (BHR; provocative concentration causing a $10 \%$ fall in the forced expiratory volume in one second (FEV1; $\mathrm{PC}_{10} \leqslant 8 \mathrm{mg} \cdot \mathrm{mL}^{-1}$ of histamine), COPD (defined as Global Initiative for Chronic Obstructive Lung Disease stage II or higher), lung function level and the longitudinal course of FEV1.

The intronic C5774T SNP of SOD2 was significantly associated with the presence of COPD and BHR in the total population. The T/T genotype for this polymorphism and the Val/Val genotype for the SOD2 Ala16Val substitution were risk factors for BHR in individuals without COPD. The SOD3 Arg213Gly substitution was associated with slower FEV 1 decline in never-smokers exclusively, and the SOD3 G(-4466)T SNP was associated with a lower vital capacity level.

Both SOD2 polymorphisms are associated with bronchial hyperresponsiveness, a risk factor for chronic obstructive pulmonary disease, while SOD2 C5774T additionally confers a risk for chronic obstructive pulmonary disease in the total population. The current authors furthermore confirm previously reported associations of SOD3 single nucleotide polymorphisms with lung function in the general population.

KEYWORDS: Bronchial hyperresponsiveness, chronic obstructive pulmonary disease, oxidative stress, single nucleotide polymorphism, superoxide dismutases

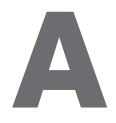

$\mathrm{n}$ imbalance between oxidants and antioxidants is considered to be an important causative factor in the onset and progression of chronic obstructive pulmonary disease (COPD) $[1,2]$. A key molecule in the regulatory process of oxidative stress is the superoxide anion, which is inhaled through cigarette smoke and additionally generated during numerous cellular reactions [3]. The free superoxide anion is a substrate for the synthesis of highly reactive oxygen species, which can damage epithelial cells, impair epithelial ciliary function, alter expression of cellular adhesion molecules, and increase airway smooth muscle contraction in response to histamine and other stimuli in both in vitro and in vivo models [4]. Therefore, impaired superoxide metabolism may contribute to the development of bronchial hyperresponsiveness (BHR) and the subsequent onset of COPD $[5,6]$. Indeed, it has previously been shown that patients with COPD have increased production of the superoxide anion by blood leukocytes, which is associated with more severe hyperresponsiveness [7]. However, this cross-sectional study did not elucidate whether the increased production of superoxide anions was the cause or consequence of BHR and COPD [7]. To date, studies on the relationship between superoxide anions and the development of both COPD and $\mathrm{BHR}$ in the general population are lacking.

The family of superoxide dismutases (SODs) is the only enzymatic system able to degrade a superoxide anion [8]. Impairment of the mitochondrial SOD (SOD2) activity was related to asthma pathophysiology [9], and extracellular

\section{AFFILIATIONS}

Depts of *Epidemiology, and

\#Pulmonology, University Medical Center Groningen, University of

Groningen, Groningen, The

Netherlands.

CORRESPONDENCE

H.M. Boezen

Dept of Epidemiology, University

Medical Center Groningen

E3.29

P0 Box 30.001

9700 RB Groningen

The Netherlands

Fax: 31503614493

E-mail: H.M.Boezen@epi.umcg.nl

Received:

December 192007

Accepted after revision:

December 162008

SUPPORT STATEMENT

This study was supported by a grant from: the Dutch Asthma Foundation (grant 3.2.02.51); University of

Groningen, Groningen, The

Netherlands; and Graduate School for Drug Exploration (GUIDE), University Medical Center Groningen, University of Groningen

STATEMENT OF INTEREST

None declared.

Online ISSN 1399-3003 
SOD (SOD3) was shown to protect lungs against oxidantmediated injury $[10,11]$. Therefore, it is of interest to investigate whether single nucleotide polymorphisms (SNPs) in these $S O D$ genes contribute to the development of BHR and/or COPD. SOD2 contains one prevalent nonsynonymous $\mathrm{SNP}$, i.e. Ala16Val, which is localised in a protein signal sequence, and thus possibly has a functional role [12-14]. This substitution has been associated with a higher lung cancer risk in Caucasians [15-17] but not with the presence of COPD in Caucasian and Chinese smokers [18, 19]. SOD3 contains three nonsynonymous SNPs, i.e. Ala40Thr, Phe131Cys and Arg213Gly. The Arg213Gly substitution has been associated with elevated SOD3 levels in human plasma [20] and with protection against COPD development in Caucasians [18, 21], whereas Ala40Thr and Phe131Cys have not yet been studied in this context. Recently, in the Copenhagen City Heart Study [22], it has been shown that the rs8192288 SNP in the SOD3 5'untranslated region is associated with lower forced vital capacity in two large Danish cohorts and constitutes a risk for more frequent COPD hospitalisation.

The present study links the well-known risk factors for COPD development, i.e. BHR and smoking, with polymorphisms located in genes involved in response to oxidative stress, a key manifestation in COPD pathogenesis. Furthermore, it aims to broaden the knowledge on candidate SOD3 SNPs with respect to (the course of) lung function and it provides new insight into the SOD2 role in this context.

\section{METHODS}

\section{Subjects}

A total of 1,390 subjects of the Vlagtwedde-Vlaardingen cohort who participated in the previous 1989-1990 survey [23] were included in the present study. This general population-based cohort of Caucasian individuals of Dutch descent started in 1965 and has been followed up for 25 yrs [23-25]. Standardised forced expiratory volume in one second (FEV1) and vital capacity (VC) measurements were performed every 3 yrs (see online supplementary material for details) [26]. The present study's protocol was approved by the local university hospital medical ethics committee and all participants gave their written informed consent.

\section{DNA extraction and genotyping}

The online supplementary material provides a description of DNA extraction and the genotyping protocol. Six SNPs in two SOD genes were genotyped, i.e. Ala16Val (rs4880) and C5774T (rs2842958) in SOD2, and Ala40Thr (rs2536512), Arg213Gly (rs1799895), G(-4466)T (rs8192288), and Phe131Cys [27] in SOD3.

The C5774T SNP was included to enable tagging of SOD2 haplotypes, as described previously [28].

\section{Presence of COPD and BHR phenotype}

The present authors identified subjects with COPD according to the Global Initiative for Chronic Obstructive Lung Disease (GOLD) criteria, i.e. an FEV1/VC $<70 \%$ and an FEV1 $<80 \%$ predicted (\% pred; GOLD stage II or higher) at the last survey [29].

Bronchial responsiveness to histamine (the 30-s method) was assessed in a random subsample of the total cohort, as described previously [25]. BHR at the last survey was expressed dichotomously, i.e. as a provocative concentration causing a $10 \%$ fall in the FEV1 (PC10) $\leqslant 8 \mathrm{mg} \cdot \mathrm{mL}^{-1}$ histamine.

\section{Statistical analysis}

Differences in prevalence of rare alleles of SNPs between subjects with and without COPD or BHR were tested using the Chi-squared test and logistic regression. Additionally, the present authors performed all mentioned analyses stratified according to smoking habits (never- and ever-smokers), as the SOD effects were expected to depend on smoking habits. For two SOD3 SNPs, it was not possible to estimate the odds ratios for COPD and BHR for the homozygous mutant genotype due either to the lack of Arg213Gly or to the low number of G(4466)T $(n=3)$ subjects.

In order to disentangle the phenotypes for COPD and BHR, subjects were classified into four phenotypic groups according to the presence of COPD and/or BHR (i.e. "COPD-/BHR-", "COPD-/BHR+", "COPD+/BHR-" and "COPD+/BHR+"). Multinomial logistic regression, adjusted for pack-yrs of smoking, was performed separately for all SNPs, with the four phenotypic groups as dependent variables. These analyses allowed prediction of whether subjects with certain genotypes were more likely to develop a COPD phenotype or a BHR phenotype.

Linear regression analysis (adjusted for sex, height, age and pack-yrs of smoking) was used to test whether the FEV1 or VC level differed between $S O D$ genotypes at the last survey [23]. Linear mixed effect models were used to estimate longitudinal changes in lung function in the total cohort, as well as in the ever- and never-smokers subgroups (see online supplementary material for details) [30].

Haplotype analysis was performed (see online supplementary material for details) [31, 32]. The remaining statistical analyses were performed and $\mathrm{p}$-values $<0.05$ were considered to be significant (using two-tailed tests).

\section{RESULTS}

\section{Study population and distribution of SOD SNPS}

The study population characteristics and genotype frequencies are shown in table 1 . All SNPs were distributed according to the Hardy-Weinberg equilibrium in the total population and were not correlated with each other $\left(\mathrm{r}^{2}<0.27\right.$ for any SNP pair). The Phe131Cys substitution in SOD3 was not present in the current cohort. No homozygote mutants were found for the Arg213Gly SNP in SOD3 in the present population. Three and five haplotypes were identified in SOD2 and SOD3, respectively, occurring with a frequency $>1 \%$ in the total population (table S4 in the online supplementary material).

\section{SOD SNPS and the presence of COPD}

The SOD2 C5774T SNP was significantly associated with COPD in the total population and in ever-smokers (table 2 and table S2 in supplementary material online) but not in never-smokers (data not shown). No significant associations were found for the other investigated SNPs (table 2). The haplotype containing the SOD2 C5774T SNP was associated with significantly higher odds for the presence of COPD (recessive effect; odds ratio (OR) $2.1(95 \%$ confidence interval (CI) 1.1-4.0), $\mathrm{p}=0.02)$, compared 


\begin{tabular}{|c|c|c|}
\hline $\begin{array}{l}\text { Char } \\
\text { Vlaal }\end{array}$ & $\begin{array}{l}\text { of the sample o } \\
\text { nort investigated }\end{array}$ & $\begin{array}{l}\text { Vlagtwedde- } \\
\text { he last survey }\end{array}$ \\
\hline & Total population & Ever-smokers \\
\hline Subjects n & 1390 & 945 \\
\hline Males & 714 (51.4) & $610(64.6)$ \\
\hline Age yrs & 52 (35-79) & 50 (35-79) \\
\hline Pack-yrs of smoking & $8.0(0-262.2)$ & $18.8(0-262.2)$ \\
\hline FEV $1 \%$ pred & $92.6 \pm 15.3$ & $90.6 \pm 15.5$ \\
\hline vC \% pred & $101.8 \pm 14.5$ & $100.2 \pm 14.0$ \\
\hline FEV1/VC \% & $73.9 \pm 8.7$ & $72.4 \pm 9.2$ \\
\hline $\begin{array}{l}\text { COPD GOLD } \\
\text { stage II or over }\end{array}$ & $167(12.4)$ & $140(15.3)$ \\
\hline $\begin{array}{l}\text { BHR PC } 10 \\
\quad \leqslant 8 \mathrm{mg} \cdot \mathrm{mL}^{-1} \\
\text { histamine }\end{array}$ & $190(46.5)$ & $141(50.4)$ \\
\hline $\begin{array}{c}\text { Genotype frequency } \\
\% \text { heterozygotes/ } \\
\% \text { homozygotes } \mathrm{m}\end{array}$ & & \\
\hline SOD2 Ala16Val & 49.6/23.8 & 49.8/23.6 \\
\hline SOD2 C5774T & $29.3 / 4.5$ & $28.8 / 5.2$ \\
\hline SOD3 Ala40Thr & $43.5 / 11.9$ & $42.0 / 12.1$ \\
\hline SOD3 Arg213Gly & $3.8 / 0.0$ & $4.1 / 0.0$ \\
\hline SOD3 G(-4466)T & $8.6 / 0.2$ & $8.9 / 0.3$ \\
\hline
\end{tabular}

Data are presented as $n(\%)$, median (range) or mean \pm SD, unless otherwise indicated. FEV1: forced expiratory volume in one second; \% pred: \% predicted; VC: vital capacity; COPD: chronic obstructive pulmonary disease; GOLD: Global Initiative for Chronic Obstructive Lung Disease; BHR: bronchial hyperresponsiveness; $\mathrm{PC}_{10}$ : the histamine concentration causing an $\mathrm{FEV}_{1}$ decrease of $\geqslant 10 \%$ from baseline. ${ }^{\#}$ : subpopulation of the total population. ": BHR test performed on a random subgroup $(n=409)$ of the total population $(n=1,390)$.

with the wild type haplotype (table S4 in the online supplementary material).

\section{SOD SNPS and the presence of BHR}

The SOD2 C5774T SNP was significantly associated with the presence of $\mathrm{BHR}$ in the random subgroup of the total population ( $n=409 / 1,390$; table 2 and table S3 in online supplementary material). Mutant homozygotes had a significantly higher risk of having BHR compared with wild types. The Ala16Val substitution in SOD2 was borderline significantly associated with BHR ( $\mathrm{p}=0.07$ for mutant versus wild type homozygotes; table 2). Stratified analyses according to smoking showed no significant associations for these two SNPs. The haplotype containing the mutant allele of C5774T SNP was associated with an increased risk for BHR, as compared with the wild type haplotype (additive effect; OR 1.7 (95\% CI 1.1-2.6), p=0.01; table S4 in the online supplementary material).

The Ala40Thr SNP was significantly associated with BHR only in never-smokers (OR (95\% CI) for the presence of BHR Ala/ Thr versus Ala/Ala 2.84 (1.08-7.48), and for Thr/Thr versus Ala/Ala 14.40 (2.92-71.08) adjusted for FEV1\% pred), but not in the total population and not in ever-smokers (table 2).

\section{SOD SNPs and the presence of COPD and/or BHR phenotype}

A total of 213 subjects had neither COPD nor BHR (COPD-/ BHR-), 154 subjects had COPD-/BHR+, six subjects had COPD+/BHR-, and 36 subjects had COPD+/BHR+. Subjects with the homozygous Ala16Val substitution in SOD2 were most likely to be COPD-/BHR+ (OR 2.1 (95\% CI 1.1-3.8) for the $\mathrm{Val} / \mathrm{Val}$ variant compared with Ala/Ala), indicating that the Ala16Val substitution was predictive for the presence of BHR and not for COPD or their combination. Likewise, subjects with the homozygous mutant SOD2 C5774T SNP were most likely to have COPD-/BHR+ (3.1 (95\% CI 1.03-9.51) for the homozygous mutant variant compared with the wild type). Haplotype analysis revealed that the only haplotype containing mutant alleles of both SOD2 SNPs showed a significantly increased OR (for an additive effect, 1.7 (95\% CI 1.2-2.6); $\mathrm{p}=0.01$ ) for BHR in non-COPD subjects as compared with the wild type haplotype. The polymorphisms in the SOD3 gene were not associated with any of the four phenotypic outcome groups.

\section{SOD SNPs and the level of FEV1 or VC}

No significant differences between the $S O D$ genotypes in relation to the level of FEV1 or VC were observed in the total population (see table S5 in the online supplementary material) or in the never- or ever-smoker subgroups (data not shown). Subjects homozygous for the G(-4466)T SNP in SOD3 had borderline significantly lower VC (regression coefficient B (SE) -532.2 mL (292.0); $p=0.068)$, but not FEV1, compared with the homozygous wild-type group (fig. 1). There were no homozygous SOD3 G(-4466)T SNP subjects in the never-smokers. In ever-smokers that were homozygous for the SOD3 G(-4466)T SNP, the current authors observed an effect size (i.e. $-533.2 \mathrm{~mL}$ (301.2), $\mathrm{p}=0.077$ ) that was similar to that in the total cohort.

\section{SOD SNPs and longitudinal change in FEV1}

The annual FEV1 decline was similar in all genotype subgroups of the investigated SNPs (see table S6 in the online supplementary material). Stratification according to smoking habits revealed that the SOD3 Arg213Gly substitution was associated with slower FEV1 decline in never-smokers, e.g. $9.3 \mathrm{~mL}$ less decline per yr in carriers of the Arg/Gly genotype compared with the wild type (fig. 2). The other SNPs were not associated with excess FEV1 decline in stratified analyses.

\section{DISCUSSION}

Of all human tissues, the lungs are most directly and particularly exposed to noxious free radicals. Tobacco smoke and environmental air pollution are the major sources of these particles. Therefore, the redox balance regulation can be an important factor for the development of BHR, excess decline in lung function and development of COPD. The current authors have shown that SOD2, containing the mutant allele of the C5774T SNP, is a risk factor for BHR in the general population. Exclusively in subjects who had smoked, COPD was more prevalent in carriers of two copies of this mutant allele compared with wild type carriers. The SOD3 Arg213Gly substitution that has previously been shown to be protective for COPD [18, 21], was associated with slower FEV1 decline in never-smokers only. Additionally, the current study confirms a 


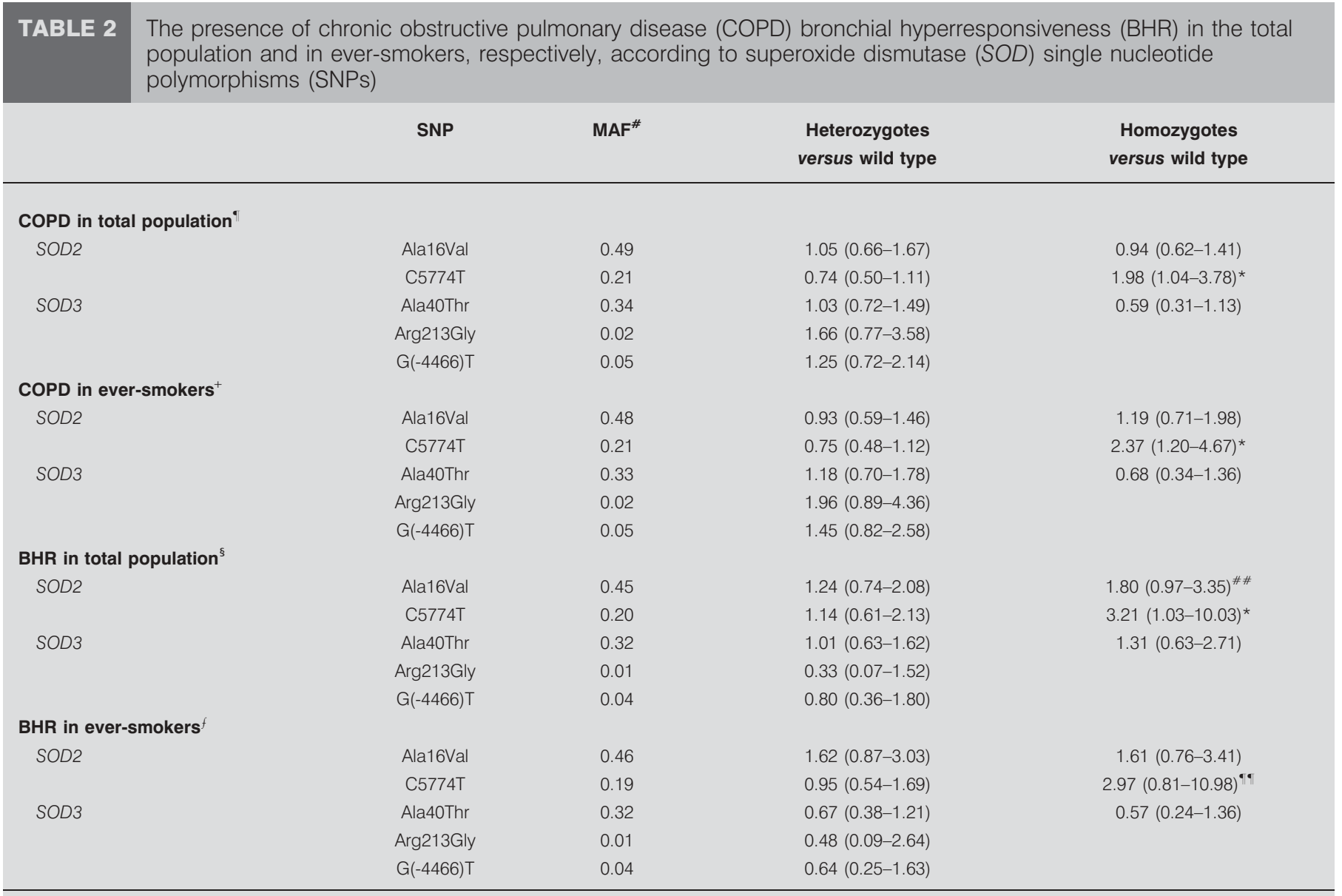

Data are presented as odds ratios (95\% confidence interval), unless otherwise indicated. Logistic regression analysis with odds ratio for COPD is adjusted for pack-yrs, and odds ratio for BHR is adjusted for pack-yrs and forced expiratory volume in one second \% predicted. MAF: minor allele frequency in the investigated group. ${ }^{\#}:$ MAF is given as a ratio of the number of the minor alleles to the number of all alleles of a certain SNP in the investigated population; ${ }^{\circ}: n=1,390 ;{ }^{+}: n=945 ;{ }^{\S}: n=409 ;{ }^{*}: n=280$. *: $p<0.05$ values are considered to be significant; ${ }^{\# \#:}: p=0.07 ; "$ " $: p=0.10$.

recently observed negative association of the G(-4466)T SOD3 SNP with lung VC level in the general population [22].

The present finding is of interest, as SOD2 dysfunction and inactivation has been recently described ex vivo in bronchial epithelial cells derived from airways of asthmatic patients [9]. This confirms the importance of SOD2 in lung homeostasis, where reactive oxygen species can impair epithelial ciliary function and increase airway smooth muscle contraction in response to histamine [4], which may contribute to the development of BHR. The present data suggest that this may also be the case in COPD.

At the last survey [23], it was found that the mutant homozygosity of the SOD2 SNP (C5774T), located in intron 3, is a risk factor for the presence of COPD and BHR in the total population. Although the role of SNPs localised in introns is not clear yet, it is known that they may affect alternative splicing processes $[33,34]$, which result in different amino acid sequences in mature proteins. The main difference between the SOD2 isoforms is the presence of an additional exon within the third intron [35], but the implication with respect to putative differences in activity between both isoforms is as yet unknown. It is indeed confirmed that the formation SOD2 isoforms may depend on genetic variations, as this has been shown in vitro for a polymorphism (single base-pair deletion) that is prevalent in African-Americans [36]. However, that specific study [36] concluded that other (cis-acting)factors are necessary for switching between two isoforms in vivo. Thus it is also possible that C5774T SNP is in high linkage disequilibrium with other functional SNPs, which are actually responsible for the associations presently observed.

Theoretically, the Ala16Val substitution could also be of importance, as in vitro studies have shown impaired transport of Val16-SOD2 to the mitochondrion [12, 13], and lower mitochondrial SOD2 activity in leukocytes for the Val/Val genotype as compared with the other genotypes [14]. An association of the Ala16Val SNP was not found neither with the presence of COPD nor with lung function decline, which is in concordance with results of two previous studies performed $[18,19]$.

Bronchial responsiveness may be modulated by superoxide anion production [7] and BHR is a known risk factor for COPD development $[5,6]$. Therefore, impaired superoxide radical 

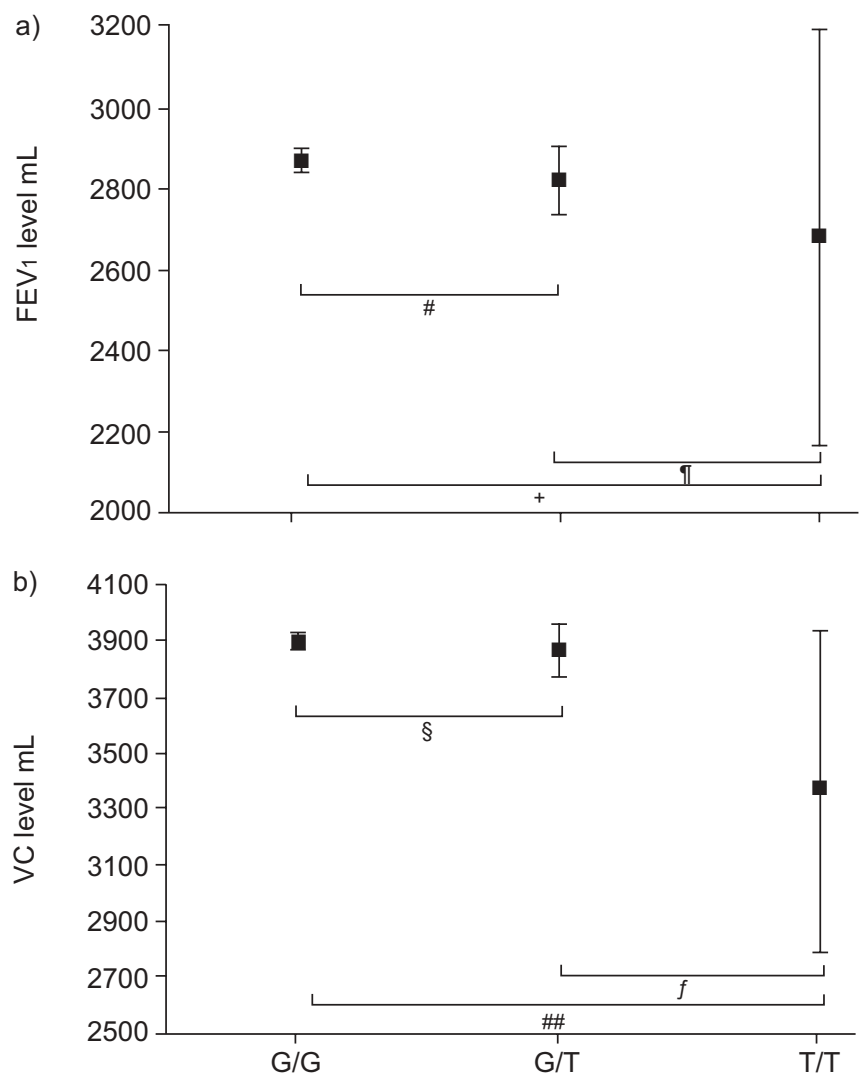

FIGURE 1. Adjusted means of a) the level of forced expiratory volume in one second (FEV1) and b) vital capacity (VC) according to the superoxide dismutase 3 $\mathrm{G}(-4466) \mathrm{T}$ genotypes in the total population. Boxes and whiskers present means and confidence intervals, respectively. ${ }^{\#}: p=0.21 ;{ }^{\top}: p=0.60 ;{ }^{+}: p=0.46 ;{ }^{5}: p=0.50$; $f: p=0.09 ;{ }^{\# \#}: p=0.07 . G / G$ subjects $n=1,236, G / T n=119$ and $T / T n=3$.

detoxification, caused by loss of SOD2 function, may increase the level of BHR and affect the development of COPD. Remarkably, subjects with homozygous variants of the SOD2 Ala16Val substitution or the SOD2 C5774T SNP showed increased ORs for having "pure BHR", which suggests it is indeed the phenotype BHR that is associated with these SOD2 SNPs, and the relationship that the present authors have found between C5774T SNP and COPD is driven by the association with BHR. Out of two mutant SOD2 haplotypes, only the one containing the mutant allele of the C5774T SNP was associated with increased risk for BHR as compared with the wild type haplotype, which implies that this SNP, rather than Ala16Val substitution, is associated with the presence of BHR.

JuUL et al. [21] have shown both prospectively and crosssectionally a lower risk for COPD for the SOD3 Arg213Gly SNP heterozygotes compared with the wild type. This has been confirmed by another cross-sectional study [18]. The underlying mechanism of these associations may be explained by reduced proteolytic processing of mutated SOD3 protein [37] and a higher SOD3 level in blood plasma due to lower affinity to heparan sulphate in the extracellular matrix [20,38]. The present authors additionally found that the protective effect of this SNP on longitudinal FEV1 change appears in neversmokers. Therefore, the present authors hypothesise that there are other antioxidant-related genetic factors that additionally

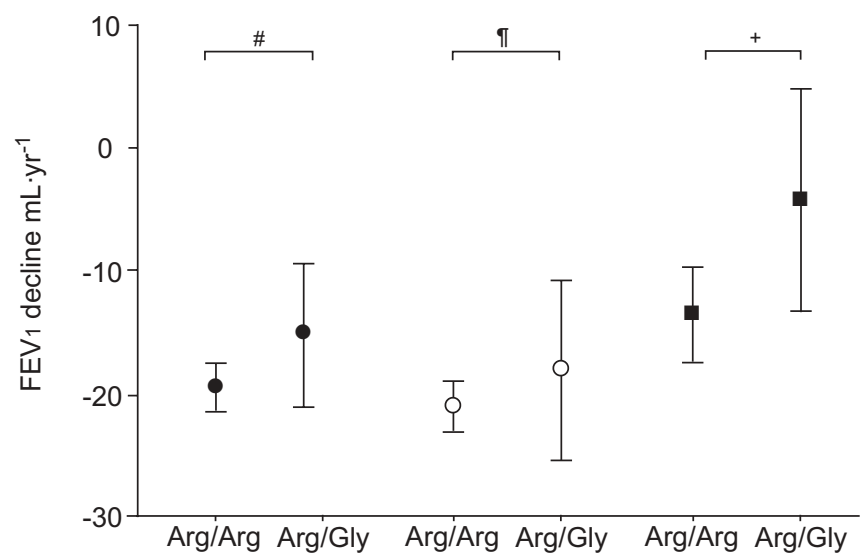

FIGURE 2. Adjusted means for the annual change in forced expiratory volume in one second (FEV1) for the superoxide dismutase (SOD)3 Arg213Gly genotypes in the total population and according to smoking habits. Boxes and whiskers present means and confidence intervals, respectively. - : total cohort; $\bigcirc$ : ever-smokers; ם : never-smokers. ${ }^{*}: p=0.14 ;{ }^{\bullet}: p=0.45 ;^{+}: p=0.03$. G/G subjects $n=1,236, G / T$ $\mathrm{n}=119$ and $\mathrm{T} / \mathrm{T} \mathrm{n}=3$.

contribute to the FEV1 change in smokers. Recently, in the Copenhagen City Heart Study, DAHL et al. [22] reported that the G(-4466)T (rs8192288) SNP, and particularly the homozygote mutant genotype, in SOD3 constitutes a risk factor for a low level of forced vital capacity and more frequent COPD hospitalisation. Interestingly, in the present study, the same SNP was borderline significantly associated with low lung VC level, with a relatively large effect size. However, only three subjects were identified as carrying the risk (homozygous mutant) genotype and therefore it was impossible to either detect likely associations with FEV1 decline or estimate the OR for COPD presence.

There are some limitations to the present study. It is conceivable that the low prevalence of the C5774T SNP in SOD2 and especially of G(-4466)T and Arg213Gly in SOD3 may have negatively affected the power of the present study. Therefore, associations with BHR, FEV1 decline or with COPD in the general population or in ever-smokers may have been impossible to detect. Another study limitation is the fact that the Vlagtwedde-Vlaardingen cohort is a unique prospective study with measurements of both BHR and FEV1 at several timepoints over 25 yrs. Therefore, it is not possible to check for replication of the present findings in another prospective cohort in order to reduce type I error (false positives). Additionally, p-values were not corrected for multiple comparisons.

In summary, it can be concluded that superoxide dismutase 2 is an antioxidant enzyme in which variations in DNA sequence can play a role in the development of bronchial hyperresponsiveness. By showing the protective effect of the Arg213Gly single nucleotide polymorphism in SOD3 on the course of forced expiratory volume in one second in never-smokers, another piece of the puzzle of the role of this substitution in pulmonary disease is added. Additionally, supportive evidence for an association of G(-4466)T (rs8192288) SOD3 single nucleotide polymorphism with the level of vital capacity in the general population is provided. Further studies are needed to confirm the possible role of $S O D$ single nucleotide 
polymorphisms in chronic obstructive pulmonary disease and bronchial hyperresponsiveness, and additional functional studies are warranted.

\section{REFERENCES}

1 Kinnula VL. Focus on antioxidant enzymes and antioxidant strategies in smoking related airway diseases. Thorax 2005; 60: 693-700.

2 Domej W, Foldes-Papp Z, Flogel E, Haditsch B. Chronic obstructive pulmonary disease and oxidative stress. Curr Pharm Biotechnol 2006; 7: 117-123.

3 Tsuchiya M, Thompson DF, Suzuki YJ, Cross CE, Packer L. Superoxide formed from cigarette smoke impairs polymorphonuclear leukocyte active oxygen generation activity. Arch Biochem Biophys 1992; 299: 30-37.

4 Henricks PA, Nijkamp FP. Reactive oxygen species as mediators in asthma. Pulm Pharmacol Ther 2001; 14: 409420.

5 Postma DS, Boezen HM. Rationale for the Dutch hypothesis. Allergy and airway hyperresponsiveness as genetic factors and their interaction with environment in the development of asthma and COPD. Chest 2004; 126: Suppl. 2, 96S-104S.

6 Brutsche $\mathrm{MH}$, Downs $\mathrm{SH}$, Schindler C, et al. Bronchial hyperresponsiveness and the development of asthma and COPD in asymptomatic individuals: SAPALDIA cohort study. Thorax 2006; 61: 671-677.

7 Postma DS, Renkema TE, Noordhoek JA, Faber H, Sluiter HJ, Kauffman H. Association between nonspecific bronchial hyperreactivity and superoxide anion production by polymorphonuclear leukocytes in chronic air-flow obstruction. Am Rev Respir Dis 1988; 137: 57-61.

8 Fridovich I. Superoxide anion radical $\left(\mathrm{O}_{2}^{-} \cdot\right)$, superoxide dismutases, and related matters. J Biol Chem 1997; 272: 18515-18517.

9 Comhair SA, Xu W, Ghosh S, et al. Superoxide dismutase inactivation in pathophysiology of asthmatic airway remodeling and reactivity. Am J Pathol 2005; 166: 663-674.

10 Carlsson LM, Jonsson J, Edlund T, Marklund SL. Mice lacking extracellular superoxide dismutase are more sensitive to hyperoxia. Proc Natl Acad Sci USA 1995; 92: 6264-6268.

11 Folz RJ, Abushamaa AM, Suliman HB. Extracellular superoxide dismutase in the airways of transgenic mice reduces inflammation and attenuates lung toxicity following hyperoxia. J Clin Invest 1999; 103: 1055-1066.

12 Sutton A, Khoury H, Prip-Buus C, Cepanec C, Pessayre D, Degoul F. The Ala16Val genetic dimorphism modulates the import of human manganese superoxide dismutase into rat liver mitochondria. Pharmacogenetics 2003; 13: 145157.

13 Sutton A, Imbert A, Igoudjil A, et al. The manganese superoxide dismutase Ala16Val dimorphism modulates both mitochondrial import and mRNA stability. Pharmacogenet Genomics 2005; 15: 311-319.

14 Fujimoto H, Taguchi J, Imai Y, et al. Manganese superoxide dismutase polymorphism affects the oxidized low-density lipoprotein-induced apoptosis of macrophages and coronary artery disease. Eur Heart J 2008; 29: 1267-1274.
15 Liu G, Zhou W, Wang LI, et al. MPO and SOD2 polymorphisms, gender, and the risk of non-small cell lung carcinoma. Cancer Lett 2004; 214: 69-79.

16 Wang LI, Miller DP, Sai Y, et al. Manganese superoxide dismutase alanine-to-valine polymorphism at codon 16 and lung cancer risk. J Natl Cancer Inst 2001; 93: 1818-1821.

17 Wang LI, Neuberg D, Christiani DC. Asbestos exposure, manganese superoxide dismutase (MnSOD) genotype, and lung cancer risk. J Occup Environ Med 2004; 46: 556-564.

18 Young RP, Hopkins R, Black PN, et al. Functional variants of antioxidant genes in smokers with COPD and in those with normal lung function. Thorax 2006; 61: 394-399.

19 Mak JC, Ho SP, Yu WC, et al. Polymorphisms and functional activity in superoxide dismutase and catalase genes in smokers with COPD. Eur Respir J 2007; 30: 684690.

20 Sandstrom J, Nilsson P, Karlsson K, Marklund SL. 10-fold increase in human plasma extracellular superoxide dismutase content caused by a mutation in heparin-binding domain. J Biol Chem 1994; 269: 19163-19166.

21 Juul K, Tybjaerg-Hansen A, Marklund S, Lange $P$, Nordestgaard BG. Genetically increased antioxidative protection and decreased chronic obstructive pulmonary disease. Am J Respir Crit Care Med 2006; 173: 858-864.

22 Dahl M, Bowler RP, Juul K, Crapo JD, Levy S, Nordestgaard BG. Superoxide dismutase 3 polymorphism associated with reduced lung function in two large populations. Am J Respir Crit Care Med 2008; 178: 906-912.

23 van Diemen CC, Postma DS, Vonk JM, Bruinenberg M, Schouten JP, Boezen HM. A disintegrin and metalloprotease 33 polymorphisms and lung function decline in the general population. Am J Respir Crit Care Med 2005; 172: 329-333.

24 van Diemen CC, Postma DS, Vonk JM, Bruinenberg M, Nolte IM, Boezen HM. Decorin and TGF-beta1 polymorphisms and development of COPD in a general population. Respir Res 2006; 7: 89.

25 Rijcken B, Schouten JP, Mensinga TT, Weiss ST, De VK, Van der LR. Factors associated with bronchial responsiveness to histamine in a population sample of adults. Am Rev Respir Dis 1993; 147: 1447-1453.

26 Quanjer PH, Tammeling GJ, Cotes JE, Pedersen OF, Peslin R, Yernault JC. Lung volumes and forced ventilatory flows. Report Working Party Standardization of Lung Function Tests, European Community for Steel and Coal. Official Statement of the European Respiratory Society. Eur Respir J 1993; 6: Suppl. 16, 5-40.

27 Campo S, Sardo AM, Campo GM, et al. Extracellular superoxide dismutase (EC-SOD) gene mutations screening in a sample of Mediterranean population. Mutat Res 2005; 578: 143-148.

28 Cebrian A, Pharoah PD, Ahmed S, et al. Tagging singlenucleotide polymorphisms in antioxidant defense enzymes and susceptibility to breast cancer. Cancer Res 2006; 66: 1225-1233.

29 Global Strategy for the Diagnosis, Management and Prevention of COPD, Global Initiative for Chronic Obstructive Lung Disease (GOLD), 2006. www.goldcopd. org Date last updated: November 2008. Date last accessed: March 3, 2009. 
30 R Development Core Team (2007). R: A language and environment for statistical computing. $\mathrm{R}$ Foundation for Statistical Computing, Vienna, Austria. www.R-project.org Date last updated: December 2008. Date last accessed: March 3, 2009.

31 Lin DY, Zeng D, Millikan R. Maximum likelihood estimation of haplotype effects and haplotype-environment interactions in association studies. Genet Epidemiol 2005; 29: 299-312.

32 Zeng D, Lin DY, Avery CL, North KE, Bray MS. Efficient semiparametric estimation of haplotype-disease associations in case-cohort and nested case-control studies. Biostatistics 2006; 7: 486-502.

33 von Ahsen N, Oellerich $M$. The intronic prothrombin 19911A $>$ G polymorphism influences splicing efficiency and modulates effects of the $20210 \mathrm{G}>\mathrm{A}$ polymorphism on mRNA amount and expression in a stable reporter gene assay system. Blood 2004; 103: 586-593.
34 Kuehl P, Zhang J, Lin Y, et al. Sequence diversity in CYP3A promoters and characterization of the genetic basis of polymorphic CYP3A5 expression. Nat Genet 2001; 27: 383391.

35 Hubbard T, Barker D, Birney E, et al. The Ensembl genome database project. Nucleic Acids Res 2002; 30: 38-41.

36 Shao J, Chen L, Marrs B, et al. SOD2 polymorphisms: unmasking the effect of polymorphism on splicing. BMC Med Genet 2007; 8: 7.

37 Bowler RP, Nicks M, Olsen DA, et al. Furin proteolytically processes the heparin-binding region of extracellular superoxide dismutase. J Biol Chem 2002; 277: 16505-16511.

38 Folz RJ, Peno-Green L, Crapo JD. Identification of a homozygous missense mutation (Arg to Gly) in the critical binding region of the human EC-SOD gene (SOD3) and its association with dramatically increased serum enzyme levels. Hum Mol Genet 1994; 3: 2251-2254. 